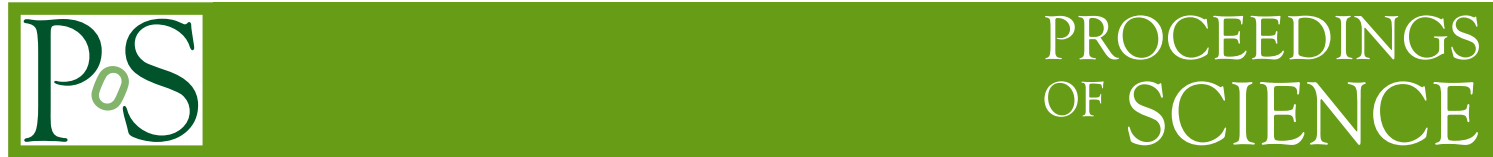

\title{
In Memory of Al Cameron
}

\section{John J. Cowan*}

Homer L. Dodge Dept. of Physics, University of Oklahoma Norman, OK 73019

E-mail: cowan@nhn.ou.edu

\section{James W. Truran}

Department of Astronomy and Astrophysics, University of Chicago, Chicago, IL 60637 and

Argonne National Laboratory, Argonne, IL 60439

E-mail: truran@nova.uchicago.edu

$\mathrm{Al}$ Cameron, who died recently (October 3, 2005) at 80, was one of the giants in astrophysics. His insights were profound and his interests were wide-ranging. Originally trained as a nuclear physicist, he made major contributions in a number of fields, including nuclear reactions in stars, nucleosynthesis, the abundances of the elements in the Solar System, and the origin of the Solar System and the Moon. In 1957, Cameron and, independently, Burbidge, Burbidge, Fowler and Hoyle, wrote seminal papers on nuclear astrophysics. Most of our current ideas concerning element formation in stars have followed from those two pioneering and historical works. Al also made many contributions in the field of Solar System physics. Particularly noteworthy in this regard was Cameron's work on the formation of the Moon. Al was also a good friend and mentor of young people. Al Cameron will be missed by many in the community both for his scientific contributions and for his friendship.

International Symposium on Nuclear Astrophysics - Nuclei in the Cosmos - IX

June 25-30 2006

CERN, Geneva, Switzerland

\footnotetext{
* Speaker.
} 


\section{Introduction}

Al Cameron was born June 21, 1925 in Winnipeg, Canada. During his long career he made many scientific contributions. He had enormous curiosity and was endlessly inventive and extremely prolific. In addition to his scientific contributions, Al Cameron had a distinguished career in public service including his years as the Chair of the Space Sciences Board of the National Academy of Sciences from 1976 to 1982[1, 2]. Cameron was a member of the faculty of Harvard University from 1973 until his retirement in 1999. He then joined the faculty of the Lunar and Planetary Laboratory of the University of Arizona, where he was a Senior Research Scientist.

\section{Some of His Scientific Accomplishments}

\subsection{Element Synthesis}

The papers by Cameron[3, 4] and Burbidge, Burbidge, Fowler and Hoyle[5] substantially defined the field of nucleosynthesis as we understand it today. Originally trained in nuclear physics, Al's interest in nuclear astrophysics was triggered by the observational results of Merrill[6], who detected the presence of the radioactive element technetium in the atmospheres of red-giant stars thereby indicating that technetium actually synthesized in these types of stars. This led Al to work on determining the sources of neutrons for this nucleosynthesis process in stars. He identified[7] the two sources of neutrons - the ${ }^{13} \mathrm{C}(\alpha, \mathrm{n}){ }^{16} \mathrm{O}$ and ${ }^{22} \mathrm{Ne}(\alpha, \mathrm{n})^{25} \mathrm{Mg}$ reactions - which remain today the main reactions driving slow neutron capture synthesis (the $s$-process) in red giants and massive stars, respectively.

Al contributed important discussions of carbon, neon, and oxygen thermonuclear reactions which provided critical early insights into the nature of the carbon, neon, and oxygen burning phases of core evolution of massive stars. Al also made enormous contributions to our understanding of the neutron capture processes responsible for the synthesis of most of the isotopes of nuclei in the mass range A $>70$. The distinguishing characteristics of the $s$-process and $r$-process were contained in those early works. They also identified a likely - although still not confirmed - site for $r$-process nucleosynthesis, i.e., in the neutron-rich material outside the core of an exploding Type II supernova. Al spent many years and developed many different models concerning the conditions and environments for the $r$-process $[8,9,10,11]$. Even in his later years, Al continued to try and understand how the $r$-process elements were formed and to identify the site for this nucleosynthesis process $[12,13]$.

\subsection{Explosive Nucleosynthesis of Iron Peak Nuclei}

Another significant product of Cameron's early intuition has been our understanding of the character of explosive nucleosynthesis, of its critical role in the production of the iron-peak nuclei present in nature, and of the associated prediction that nuclei of mass $\mathrm{A}=56$ should be formed predominantly as ${ }^{56} \mathrm{Ni}$ in supernova events. Early efforts to fit the iron abundance peak observed in nature were dominated by attempts to provide the best fit to an iron (nuclear statistical equilibrium)

peak centered rather on ${ }^{56} \mathrm{Fe}$ (see, e.g., [14]). Cameron recognized, however, that the burning timescale was the critical factor: when nuclear burning proceeds on a hydrodynamic (e.g., post shock) timescale, weak interaction processes cannot act to convert any appreciable fraction of 
protons to neutrons during the course of the thermonuclear burning epoch. It follows that, for matter initially dominated by self-conjugate nuclei $\left(\right.$ e.g. ${ }^{12} \mathrm{C},{ }^{16} \mathrm{O}$, and $\left.{ }^{28} \mathrm{Si}\right)$, the final products of explosive nucleosynthesis must also lie along or very near to the $\mathrm{Z}=\mathrm{N}$ line. Utilizing nuclear reaction and weak interaction rates developed by Cameron and his students (described below), this behavior was subsequently confirmed by detailed numerical calculations. These calculations[15] revealed ${ }^{56} \mathrm{Ni}$ to be the most abundant product of such explosive burning episodes with clear, and now widely recognized, implications for the powering of the light curves of supernovae.

\subsection{Solar System Abundances}

Cameron recognized the importance of a detailed understanding of the abundances of the elements in Solar System material as a guide to the nucleosynthetic processes by which they were produced. The abundance pattern itself confirmed the dominant role of nuclear processes in nucleosynthesis and displayed such regularities as the iron abundance peak (a nuclear statistical equilibrium feature) and the peaks in the heavy element region attributable to the effects of neutron shell features. His considerations of the neutron capture processes ultimately led to the detailed separation and identification of the distinct $s$-process and $r$-process isotopic components in the heavy element region published in his discussion of the abundances of the elements[16]. Cameron's breakdown of the solar abundances into the $s$-process, $r$-process, and $p$-process components, originally identified by Burbidge et al.[5], are shown in Figure 1[17]. It is significant to note that recent studies of heavy element abundances in extremely metal deficient stars $([18,19,20])$ closely fit the $r$-process pattern identified in Solar System matter by Cameron's early work. In fact our ideas on heavy element synthesis, and the assignments of the Solar System abundances into their respective $r, s$ and $p$-components, have deviated little from his in almost 50 years. Cameron and his collaborators also explored $r$-process synthesis in neutron-rich supernova ejecta in early dynamic $r$-process calculations[21].

\subsection{Nuclear Data for Astrophysics}

Cameron was also concerned with providing critical nuclear reaction rate and cross section data for for astrophysics calculations. He recognized that, while experimental studies in nuclear physics can provide many of the nuclear reaction cross sections critical to the early phases of stellar energy generation (e.g. hydrogen, helium, carbon, and oxygen burning), this was not sufficient for studies of the later phases of burning and nucleosynthesis, where hundreds of individual reactions can be involved. He pioneered the use of systematics of nuclear properties that ultimately made possible theoretical calculations of heavy element synthesis in diverse astronomical environments. Noteworthy in this regard are studies by Cameron and his students of nuclear mass formulae[22], of nuclear level densities[23, 24], of thermonuclear reaction rates[25], and of weak interaction rates[26]. The rates arising from these early studies enabled critical and defining early investigations of explosive nucleosynthesis in supernovae. A clear measure of the impact of this research in nuclear astrophysics is provided, for example, by the fact that the study of nuclear level densities remains today the basis for virtually all current calculations of thermonuclear reaction rates from nuclear systematics. 

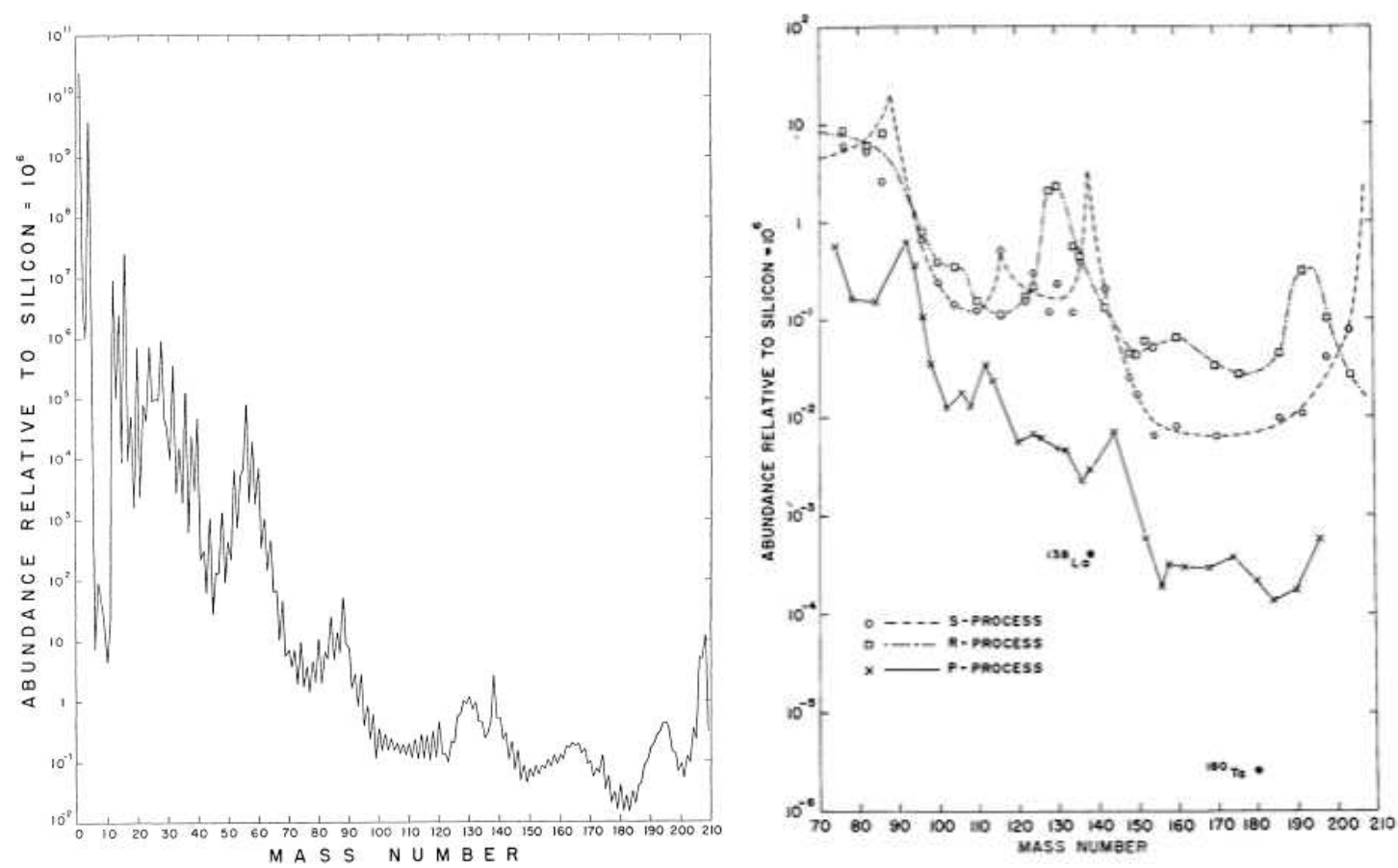

Figure 1: (left) Total and (right) components by nucleosynthetic process of the Solar System abundances from Cameron[16, 17].

\subsection{Solar System Studies and the Origin of the Moon}

Cameron also performed fundamental and pioneering work in studies of the Solar System. He developed, for example, the idea of the supernova trigger for the formation of the Solar System[27]. In this model, a single event could account for most isotopic anomalies and extinct radioactivities in the solar system material. He also spent many years trying to understand the formation of the Moon. His work established the idea of a large (Mars-sized) object impacting on the proto-Earth, ejecting matter into orbit, where it accreted to form the Moon[28]. As a result of years of numerical simulations, on a large number of distributed computers, Al's calculations demonstrated that this large-impact hypothesis could explain the masses, densities and angular momentum of the EarthMoon system.

\section{Legacies}

Al left many scientific legacies: his work on nucleosynthesis, nuclear physics input for astrophysical environments, cosmic abundances and studies of the origin of the Solar System and the Moon. Even more important $\mathrm{Al}$ was a good friend and mentor of young people. Al Cameron (1925-2005), who died in October of 2005, will be missed by many for his scientific curiosity and creativity, his sense of humor, kindness and warmth.

\section{Acknowledgments}

We thank C. Sneden for helpful comments. The work of the authors has been supported in part 

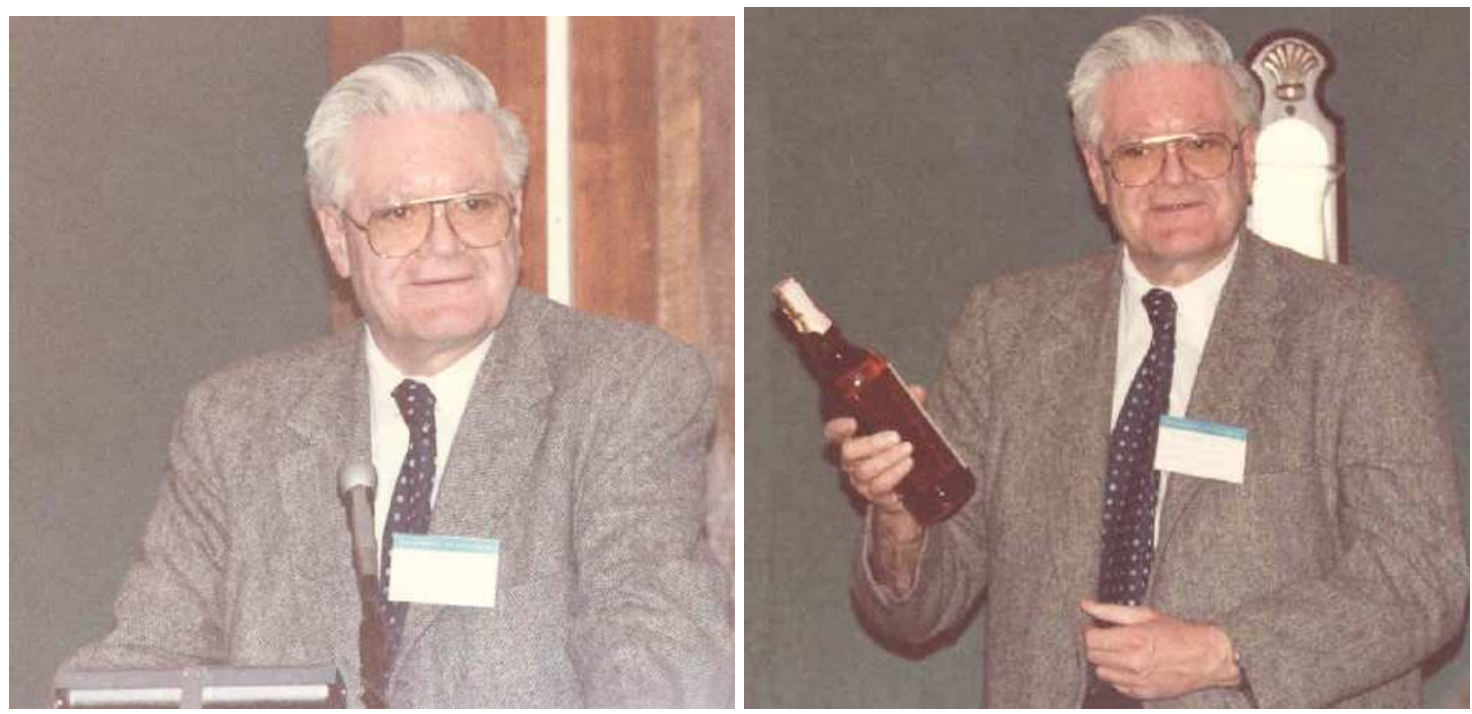

Figure 2: $\mathrm{Al}$ at work and play at a meeting in Boulder, $\mathrm{CO}$ in 1985 to celebrate his 60th birthday (see [29]).

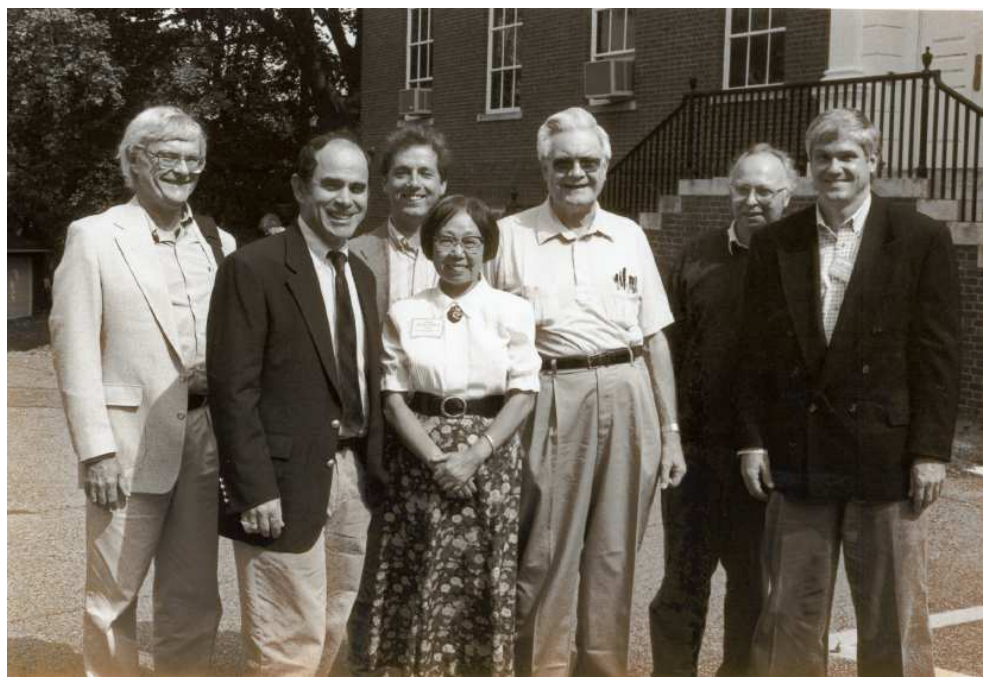

Figure 3: Al with some of his former students and postdocs in September of 1995 to celebrate his 70th birthday at the Harvard-Smithsonian Center for Astrophysics, in Cambridge, MA.

by the NSF, DOE and by STScI.

\section{References}

[1] Truran, J. W., Thielemann, F.-K, \& Cowan, J. J. Obituary: Alastair Graham Walker Cameron, 1925-2005. Bulletin of the American Astronomical Society 37, 1547-1548 (2005).

[2] Wasserburg, G. J. Obituary for Al Cameron. Physics Today 59, 68-70 (2006).

[3] Cameron, A.G.W. Nuclear reactions in stars and nucleosynthesis. Pub. Astron. Soc. Pacific 69, 201-222 (1957). 


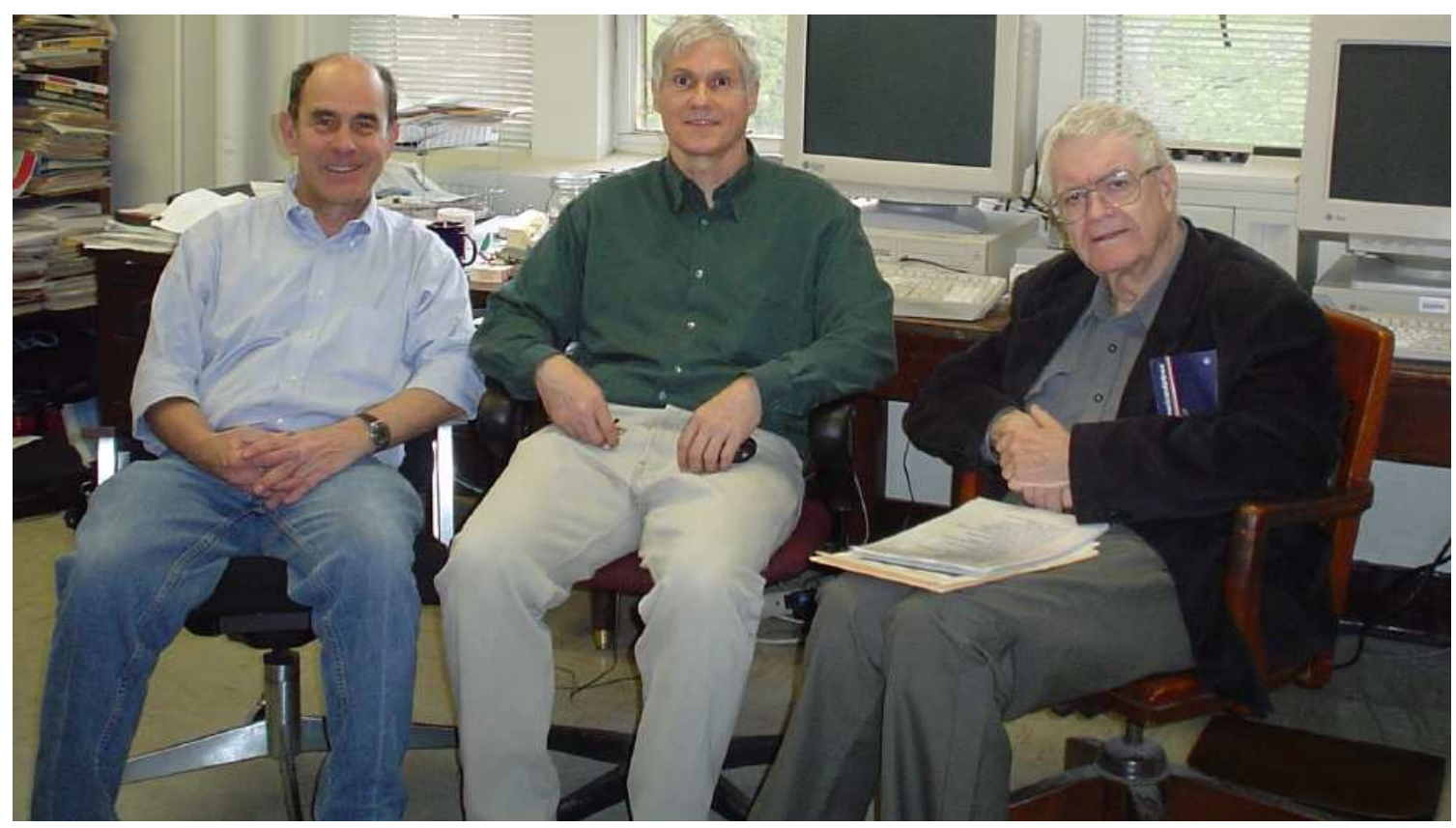

Figure 4: $\mathrm{Al}$ - still working on the $r$-process and just shortly before giving an 80 minute colloquium - with the authors in Norman, OK (April, 2005).

[4] Cameron, A.G.W. On the origin of the heavy elements. Astron. J. 62, 9 (1957).

[5] Burbidge, E.M., Burbidge, G.R., Fowler, W.A., \& Hoyle, F. Synthesis of the elements in stars. Rev. Mod. Physics 29, 547-650 (1957).

[6] Merrill, P. W. Spectroscopic observations of stars of class S. Astrophys. J. 116 21-26 (1952).

[7] Cameron, A.G.W. Origin of anomalous abundances of the elements in giant stars. Astrophys. J. 121, 144-160 (1955).

[8] Truran, J. W., Cowan, J. J., \& Cameron, A. G. W. The helium-driven r-process in supernovae. Astrophys. J. 222, L63-L67 (1978).

[9] Cowan, J. J., Cameron, A. G. W., \& Truran, J. W. Explosive helium burning in supernovae - A source of r-process elements. Astrophys. J. 265, 429-442 (1983).

[10] Cameron, A. G. W., Cowan, J. J., \& Truran, J. W. The waiting point approximation in R-process calculations. Astrophysics and Space Science 91, 235-243 (1983).

[11] Cowan, J. J., Cameron, A. G. W., \& Truran, J. W. R-process nucleosynthesis in dynamic helium-burning environments. Astrophys. J. 294, 656-662 (1985).

[12] Cameron, A.G.W. Some properties of r-process accretion disks and jets. Astrophys. J. 562, 456-469 (2001).

[13] Cameron, A.G.W. Some nucleosynthesis effects associated with r-process jets. Astrophys. J. 587, 327-340 (2003).

[14] Hoyle, F. \& Fowler, W.A. Nucleosynthesis in supernovae. Astrophys. J. 132, 565-590 (1960).

[15] Truran, J.W., Arnett, W.D., \& Cameron, A.G.W. Nucleosynthesis in supernova shock waves. Canadian Journal of Physics 45, 2315 (1967). 


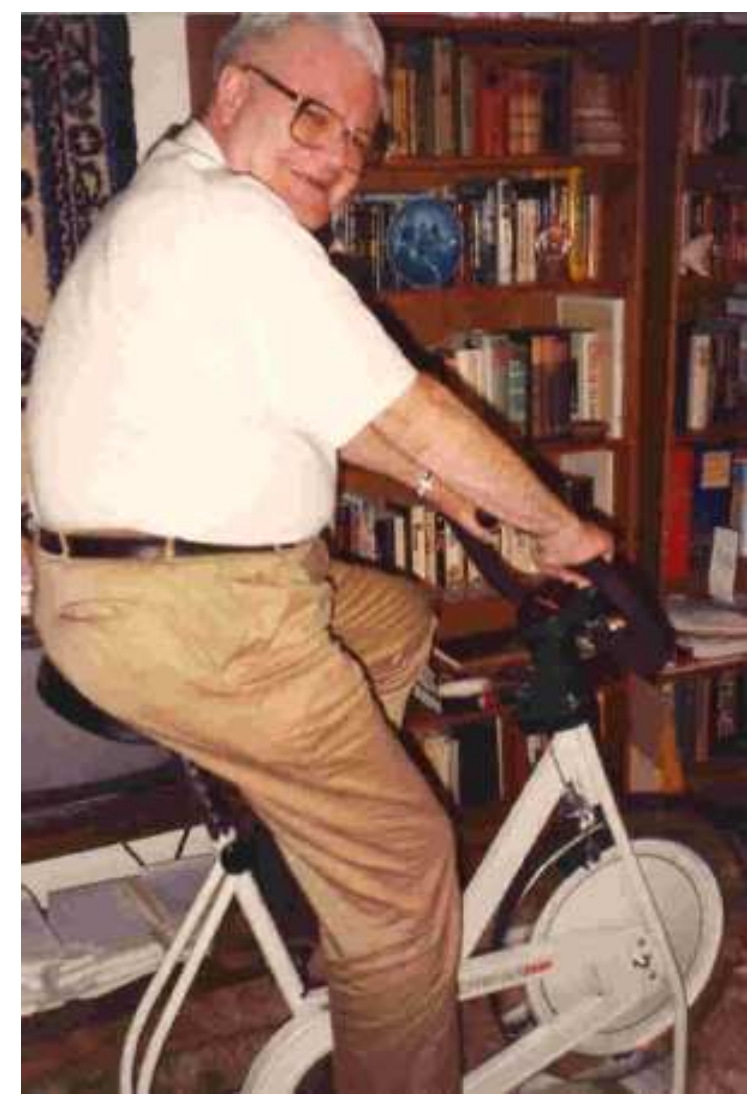

Figure 5: The only known picture of Al Cameron ever exercising taken in Norman, OK - showing Al's spirit of adventure and willingness to try anything, even exercise, at least once.

[16] Cameron, A.G.W. A Revised table of abundances of the elements. Astrophys. J. 129, 676-699 (1959).

[17] Cameron, A.G.W. Abundances of the elements in the Solar System. Space Science Reviews, 15, 121-146 (1973).

[18] Truran, J. W., Cowan, J. J., Pilachowski, C. A. \& Sneden, C. Probing the neutron-capture nucleosynthesis history of galactic matter. Pub. Astr. Soc. Pac. 114, 1293-1308 (2002).

[19] Sneden, C. \& Cowan, J. J. The genesis of the heaviest elements in the Milky Way galaxy. Science 299, 70-75 (2003).

[20] Cowan, J. J. \& Sneden, C. Heavy element synthesis in the oldest stars and the early universe. Nature 440, 1151 (2006).

[21] Delano, M.D. \& Cameron, A.G.W. Nucleosynthesis in neutron rich supernova ejecta. Astrophysics and Space Science 10, 203-226 (1971).

[22] Cameron, A.G.W. \& Elkin, R. Role of the symmetry energy in atomic mass formulas. Canadian Journal of Physics 43, 1288 (1965).

[23] Cameron, A.G.W. Nuclear level spacings. Canadian Journal of Physics 36, 1040 (1958).

[24] Gilbert, A. \& Cameron, A.G.W. A composite nuclear-level density formula with shell corrections. Canadian Journal of Physics 43, 1446 (1965). 
[25] Truran, J.W., Hansen, C.J., Cameron, A.G.W., \& Gilbert, A. Thermonuclear reactions in medium and heavy nuclei. Canadian Journal of Physics 44, 151 (1966).

[26] Hansen, C.J. Some weak interaction processes in highly evolved stars. Astrophysics and Space Science 1, 499-512 (1968).

[27] Cameron, A.G.W., \& Truran, J. W. The supernova trigger for formation of the solar system. Icarus 30, 447-461 (1977).

[28] Benz, W., Slattery, W. L., \& Cameron, A.G.W. The origin of the moon and the single-impact hypothesis I. Icarus 66, 515-535 (1986).

[29] Cameron, A.G.W. Some Autobiographical Notes in Cosmogonical Processes, eds., W.D. Arnett, C.J. Hansen, J.W. Truran, \& S. Tsuruta (Utrecht, The Netherlands; VNU Science Press), 1-31 (1986). 